\title{
Evolving treatment paradigms in esophageal cancer
}

\author{
Ilit Turgeman ${ }^{1}$, Irit Ben-Aharon ${ }^{1,2}$ \\ ${ }^{1}$ Division of Oncology, Rambam Health Care Campus, Haifa, Israel; ${ }^{2}$ Faculty of Medicine, Technion-Israel Institute of Technology, Haifa, Israel \\ Contributions: (I) Conception and design: Both authors; (II) Administrative support: I Ben-Aharon; (III) Provision of study materials or patients: \\ I Turgeman; (IV) Collection and assembly of data: I Turgeman; (V) Data analysis and interpretation: I Turgeman; (VI) Manuscript writing: Both \\ authors; (VII) Final approval of manuscript: Both authors. \\ Correspondence to: Prof. Irit Ben-Aharon, MD, PhD. Director, Division of Oncology, Rambam Health Care Campus, Haifa, Israel. \\ Email: I_Benaharon@rambam.health.gov.il.
}

\begin{abstract}
A heterogenous disease with a dismal prognosis, esophageal cancer poses a major health challenge worldwide. In recent years, the treatment landscape for esophageal adenocarcinoma and squamous cell carcinoma (SCC) has undergone major evolution, with the elucidation of underlying biologic pathways and predispositions. Neoadjuvant chemoradiation has emerged as a leading approach for the management of locoregional esophageal cancer, while perioperative chemotherapy has shown promising outcomes specifically in adenocarcinoma of the lower esophagus and gastroesophageal junction (GEJ). Studies also explore the implementation of chemoradiation in various sequential preoperative strategies, as well as in the adjuvant setting. Definitive chemoradiation is considered a valid alternative for non-surgical candidates with SCC. Clinical trials currently evaluating the potential benefits of different approaches may shed light on existing controversies regarding optimal management of locoregional disease. For patients with metastatic cancer, chemotherapy remains the backbone of antineoplastic treatment alongside palliative care, moreover the discovery of novel biological targets has led to the initiation of targeted and immune therapy for specific subpopulations. Taken together, an era of burgeoning clinical trials and changing paradigms has evolved in esophageal oncology. Multidisciplinary collaboration is key to effective combination and sequencing of treatment modalities tailored per patient and per tumor histology. This work aims to provide a comprehensive overview of state-of-the-art oncological management of esophageal cancer, with consideration of new challenges and obstacles to be overcome.
\end{abstract}

Keywords: Esophageal cancer; gastroesophageal junction (GEJ); chemoradiation; neoadjuvant; perioperative; targeted therapy; immunotherapy

Submitted Jan 13, 2020. Accepted for publication Feb 28, 2020.

doi: $10.21037 /$ atm.2020.03.110

View this article at: http://dx.doi.org/10.21037/atm.2020.03.110

\section{Introduction}

Esophageal cancer is a unique gastrointestinal malignancy in its pathophysiology, prevalence and treatment, and poses a major health challenge worldwide. Two separate malignancies arise from the esophagus: squamous cell carcinoma (SCC) and adenocarcinoma, each harboring a distinct genomic backdrop (1). The former tends to appear in the midportion of the esophagus, has a proclivity for early lymphatic spread, and, in non-endemic regions, is often associated with heavy tobacco and alcohol consumption.
The latter is generally located in the distal esophagus and gastroesophageal junction (GEJ), has overlapping characteristics with gastric cancer, and may be induced by chronic gastroesophageal reflux disease (2).

Incidence varies widely by geographic region, with disproportionate frequency in Eastern Asia as well as Southern and Eastern Africa; lower rates are reported in Europe and North America, and rarely in Northern and Western Africa and Central America (3). SCC predominates globally, while adenocarcinoma rates are rapidly rising in Western countries. Esophageal cancer comprises roughly 3\% 

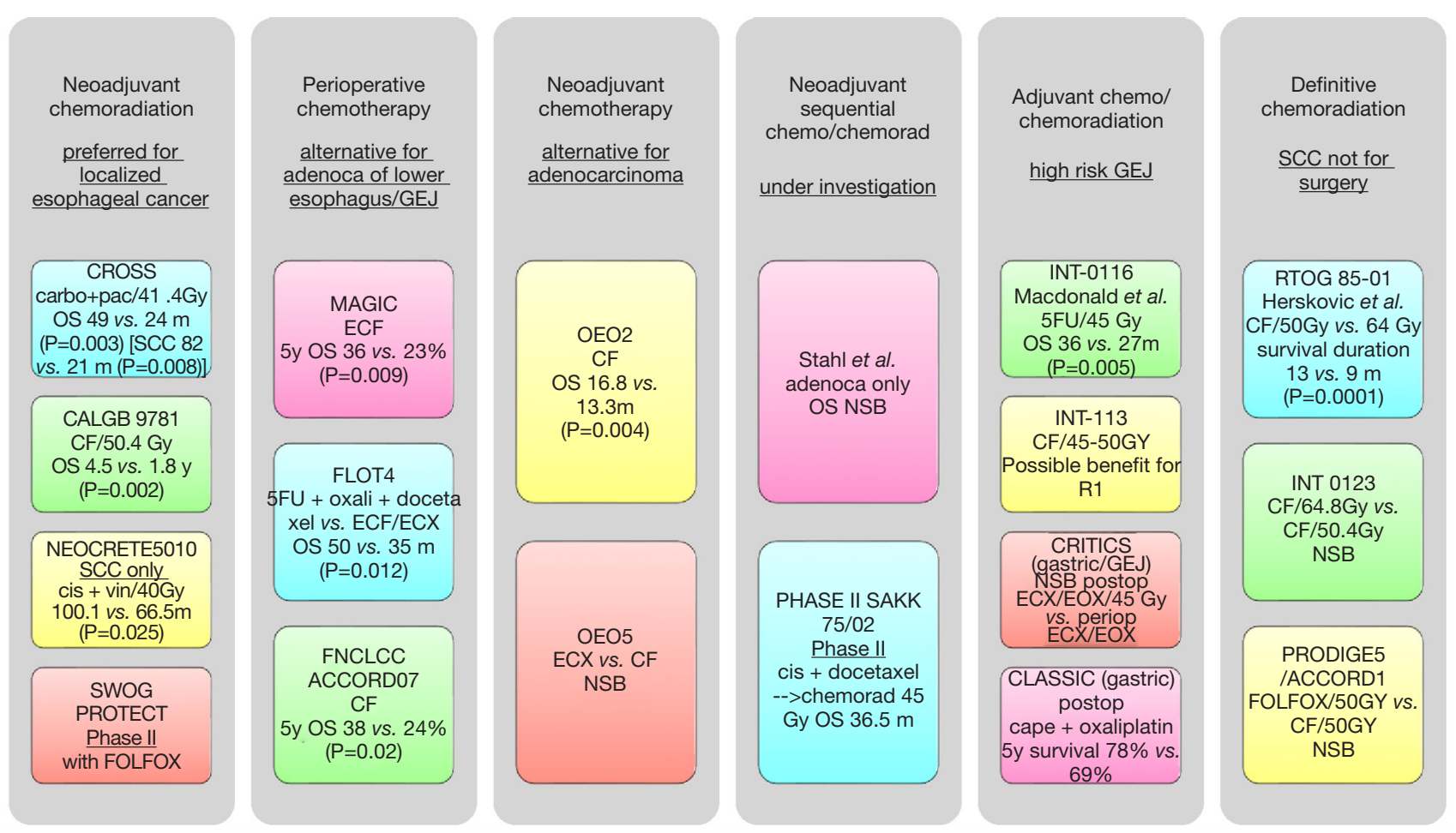

Figure 1 Landmark studies evaluating treatment approach in locoregional esophageal/GEJ cancer. Trials are phase III and enrolled patients with both adenocarcinoma and SCC unless otherwise indicated. Control arm is surgery alone unless otherwise specified. All P values listed are significant. OS, median overall survival; Chemorad, chemotherapy and radiation; Periop, perioperative; Postop, postoperative; NSB, no significant survival benefit; CF, cisplatin and fluorouracil; 5FU, fluorouracil; Cape, capecitabine; Oxali, oxaliplatin; Vin, vinorelbine; ECF, epirubicin cisplatin fluorouracil; EOX, epirubicin oxaliplatin capecitabine; ECX, epirubicin cisplatin capecitabine; m, months; y, years; GEJ, gastroesophageal junction.

of new malignancies diagnosed worldwide, nevertheless the case fatality rate approaches $90 \%$ (3) and five-year survival reaches $20 \%$ in total and $4.8 \%$ for metastatic disease (4)making esophageal cancer a disease with dismal prognosis.

In recent years, the landscape of esophageal cancer treatment has undergone major evolution. With the underlying biologic pathways and predispositions elucidated, and treatment modalities honed to target individual cancer types, a consistent yearly decrease in death rates of $0.9 \%$ has been observed (4). Multidisciplinary collaboration is the cornerstone of state-of-the-art management, in order to define optimal treatment sequencing. In this manuscript, we will comprehensively appraise current treatment paradigms for loco-regional esophageal cancer and review groundbreaking advances in metastatic disease, including the discovery of novel biological targets and use of immune checkpoint inhibitors. New challenges arising from the plethora of data and obstacles for further research will also be considered.

\section{Locoregional esophageal carcinoma: chemotherapy and radiotherapy-neoadjuvant, perioperative, adjuvant, and definitive approaches}

Roughly half of patients diagnosed with esophageal carcinoma present with localized or loco-regional disease, while in $40 \%$, cancer has spread to distant sites (the remaining $10 \%$ present with unclear staging) (4). Historically, surgery has been the standard treatment for stage I to III disease, and survival rates barely exceeded one year (5). Radiation has also been used as monotherapy for loco-regional control of esophageal cancer, and rates were comparable to surgery (6). These poor longterm outcomes provided the impetus for the advent of multimodality approaches to treat non-metastatic 
disease (Figure 1). Early T1-2N0 cancer is still effectively treated with single surgical approaches, but survival rates drop as T-stage increases or in the case of lymph node involvement (7). To date, international guidelines recommend neoadjuvant treatment for at least clinical T3 or $\mathrm{N}+$ tumors $(8,9)$. The rationale for combining chemotherapy and radiotherapy is the radio-sensitizing effect, to downstage disease and achieve local control, as well as to potentially eradicate micrometastatic disease and enhance long-term survival.

\section{Neoadjuvant chemoradiotherapy-preferred treatment paradigm for localized esophageal cancer (T3Nx)}

Neoadjuvant concurrent chemoradiation prior to curative surgery has shown significant survival advantages compared with surgery alone, regardless of histology or tumor location. Three large randomized phase III trials published positive results for tri-modality therapy in resectable esophageal carcinoma, compared with surgery alone: the Dutch CROSS and the CALGB 9781, where the majority of patients had adenocarcinoma, and the Chinese NEOCRTEC5010 trial, where only patients with SCC were permitted. Of note, CALGB 9781 was closed early due to poor accrual, but results were significant.

In the largest and practice-changing Dutch CROSS trial (10), a low-dose weekly carboplatin plus paclitaxel regimen was used concurrently with 41.4 Gy radiation, while cisplatin plus fluorouracil (5-FU)/50.4 Gy protocol was used in CALGB 9781 (11) and vinorelbine and cisplatin/ 40 Gy in the NEOCRTEC5010 trial (12). In CROSS, the microscopically complete resection (R0) rate was higher with chemoradiotherapy (92\% vs. 69\%), and $29 \%$ of those treated with chemoradiotherapy had a pathologic complete response (pCR). The rate of pCR in the SCC cohort was 49\% and in the other two trials also above $40 \%$. A persistent survival benefit was shown in all three studies compared with surgery alone. Median follow-up in the CROSS trial was 84 months, achieving median overall survival (OS) of $48.6 v s .24$ months, and a five-year survival rate of $47 \%$ versus $33 \%$ (13). The smaller group of patients with SCC had an OS of $81.6 \mathrm{vs}$. 21.1 months. Overall recurrence rate was $35 \%$ compared to $58 \%$ in the surgery alone group, further indicating that both locoregional recurrence and peritoneal carcinomatosis rate were significantly reduced. Median OS in the CALGB 9781 trial was 4.5 vs. 1.8 years, in an intent-to-treat analysis, and in NEOCRTEC5010, 100.1 vs. 66.5 months. There were no differences in postoperative morbidity or mortality rates between the two groups in all three studies.

The utility of preoperative chemoradiotherapy has been confirmed in several subsequent meta-analyses showing improved overall survival when compared to all other treatment modalities including surgery alone, neoadjuvant chemotherapy and neoadjuvant radiotherapy, however at the cost of increased postoperative mortality $(14,15)$. In a separate meta-analysis, the hazard ratio for all-cause mortality for neoadjuvant chemoradiotherapy was 0.78 , yielding an absolute survival benefit of 8.7 percent at two years and a number-needed-to-treat to prevent one death of 11. Significance was maintained across histologic subtypes, and was not offset by postoperative mortality (16). A clear advantage to neoadjuvant chemoradiotherapy over neoadjuvant chemotherapy was not established.

An acceptable alternative chemotherapy regimen to the widely used protocols described above is fluorouraciloxaliplatin-folinic acid (FOLFOX), which has shown encouraging results in several phase II trials $(17,18)$.

\section{Perioperative chemotherapy-alternative for adenocarcinoma of the lower esophagus or GEF}

Two large phase III trials have placed perioperative chemotherapy in the forefront of treatment for GEJ and gastric cancer. In the seminal Medical Research Council Adjuvant Gastric Infusion Chemotherapy MAGIC trial (19) three cycles of both pre and post-operative epirubicin/ cisplatin/5-FU (ECF) were superior to surgery alone, with a $15 \%$ improvement in OS. Subsequently, the FLOT4 trial from Germany enrolled patients with GEJ (66\%) or gastric (34\%) adenocarcinoma, and reported superior results with four cycles of perioperative infusional-5-FU, oxaliplatin and docetaxel, as compared with the MAGIC regimen; median OS was 50 vs. 35 months, five-year OS was $45 \%$ vs. $36 \%$ (20). and higher rates of $\mathrm{R} 0$ resection and lower staging at surgery were observed, with pCR rate of $16 \%$ vs. $6 \%$ (21). Toxicity was comparable in both groups. Thereafter, the three drug FLOT regimen emerged as the preferred chemotherapy regimen for patients with locally advanced carcinoma of the GEJ. However, due to considerable toxicity, FLOT should be substituted with FOLFOX in those with inferior performance status. Furthermore, perioperative chemotherapy with cisplatin and fluorouracil (CF) is another viable option for both GEJ and lower esophageal adenocarcinoma, as shown in the FNCLCC ACCORD 07 trial, which demonstrated a $38 \%$ five-year OS rate $v$ s. $24 \%$ for surgery alone (22). 


\section{Neoadjuvant chemotherapy - acceptable alternative for adenocarcinoma}

Preoperative chemotherapy is another evidence-based approach for locally advanced potentially resectable esophageal cancer, yet rarely used. In the Medical Research Council OEO2 trial, a median OS benefit of $16.8 v s$. 13.3 months was gained after preoperative CF compared to surgery alone (23), and long term follow-up confirmed a $23 \%$ vs. $17 \%$ five-year OS, respectively (24). Although treatment effect did not significantly differ by histology, the established efficacy of chemoradiation in SCC makes neoadjuvant chemotherapy a feasible approach in adenocarcinoma. Notably, triplet chemotherapy did not have an OS advantage in the OEOS trial (25).

\section{Neoadjuvant sequential chemotherapy and chemoradiation therapy}

Sequential preoperative therapy, consisting of induction chemotherapy followed by concurrent chemoradiation, is under investigation in clinical trials, but has not yet shown superiority to other treatment approaches. A phase III study in adenocarcinoma patients showed higher probability of pCR and tumor-free lymph nodes at resection, with sequential treatment as compared with preoperative chemotherapy alone, as well as a trend towards a survival advantage that did not reach statistical significance (26). Numerous phase II studies showed mixed results but in general were not superior to those observed with preoperative chemoradiation $(27,28)$. One exception is the phase II $S A K K$ 75/02 trial, where docetaxel and cisplatin were administered to patients with SCC or adenocarcinoma, followed by chemoradiation with the same regimen, and showed R0 resection in 52 of the 57 participants, as well as an OS of 36.5 months (29). This approach should be further evaluated in phase III trials.

\section{Adjuvant chemotherapy/ chemoradiotherapy for high risk GEF}

Post-operative treatment remains a valid yet rarely implemented option in locally advanced esophageal and GEJ cancer, due to widespread use of the neoadjuvant approach. The landmark Intergroup INT-0116 trial investigated postoperative chemotherapy plus chemoradiation compared with surgery alone and enrolled patients with gastric and GEJ tumors (20\%). A superior median OS of $36 v s$.
27 months was shown for combined treatment (30), which was maintained after 10 years of follow-up (31). Local and regional relapse rates were reduced. The majority of patients had advanced disease with pT3/4 tumors (69\%) and positive locoregional nodes (85\%). A limitation of this study is in the surgical technique, with a low rate of lymph node retrieval (54\% underwent only D0 lymphadenectomy and only $10 \%$ a full D2 dissection), casting doubt on the quality of local control initially achieved.

Other studies have added support for post-operative chemoradiation in specific subgroups of patients with esophageal and GEJ patients. Radiation concurrent with $\mathrm{CF}$ in poor prognosis patients showed improved OS, distant metastatic and locoregional control, in a phase II comparison with a matched historical cohort of surgeryonly patients (32). A recent meta-analysis also favored this approach rather than post-operative chemotherapy or radiotherapy alone (33). Additionally, post-operative chemoradiation may have a role in the case of involved surgical resection margins (R1), as demonstrated in long term results of the Int-113 trial, whereby adjuvant treatment was delivered following an R1 resection, and was found to enhance survival in a small percentage of patients (34). The role of adjuvant chemoradiation was addressed in the large international phase III trial, the CRITICS study, which showed no survival benefit for the addition of postoperative chemoradiotherapy to perioperative chemotherapy in patients with GEJ or gastric carcinoma (35).

Evidence for adjuvant chemotherapy only (not in the setting of perioperative treatment) is extrapolated from the CLASSIC trial, which evaluated adjuvant capecitabine and oxaliplatin in advanced gastric cancer following gastrectomy plus D2 lymph node dissection, and demonstrated improved five-year OS rate of $78 \%$, compared with $69 \%$ with surgery alone (36).

It is important to note that to date, survival benefit of post-operative chemoradiation has not been demonstrated in a randomized trial in the subpopulation of esophageal cancer. Taken together, there is evidence to employ adjuvant chemoradiation only for high risk patients with GEJ cancer who have not received preoperative therapy.

\section{Definitive chemoradiotherapy-unresectable disease or poor surgical candidates, particularly SCC}

Concurrent chemoradiotherapy has been compared to RT alone and has consistently shown superior survival indices-making concurrent treatment the current standard 
of care for inoperable disease (37). Notably, the landmark Radiation Therapy Oncology Group (RTOG) 85-01 trial enrolled patients with both SCC and adenocarcinoma and was closed prematurely due to significant survival advantage both locoregionally and distally, using CF as compared to RT with 64 Gy (38). Long term follow-up showed a five-year survival of $26 \%$ vs. $0 \%$ and median survival of $14 v s .9$ months $(39,40)$. However, an unacceptably high locoregional failure rate of nearly $50 \%$ at one year was observed and subsequently addressed in a follow-up trial, INT 0123 (41). Herein, higher doses of RT (64.8 vs. $50.4 \mathrm{~Gy}$ ) were not associated with improved survival or locoregional control and rather more toxicity incurred, validating the standard dose of 50.4 Gy; notwithstanding, this was prior to the era of modern radiotherapy techniques.

Newer randomized studies have found utility in other chemotherapeutic regimens when used as radiosensitizers. The phase II/III PRODIGE5/ACCORD17 presented promising results with concurrent FOLFOX, although survival endpoints were not superior to the control, CF (42). FOLFOX represents an effective alternative to $\mathrm{CF}$. The docetaxel and cisplatin regimen has also demonstrated efficacy in SCC patients treated with definitive chemoradiotherapy (43).

A non-operative approach should be indicated for SCC located in the cervical esophagus. In the previous SCC study, the overall response rate was $98.3 \%$ with complete response achieved in $71 \%$ of patients and a median OS of 23 months. Furthermore, as mentioned above for the CROSS trial, pCR was observed in $49 \%$ of SCCs compared with $23 \%$ of adenocarcinomas. Of note, perioperative mortality is generally higher for SCC than for adenocarcinoma, perhaps due to associated comorbidity and higher tumor location $(44,45)$. In a meta-analysis of randomized controlled studies comparing neoadjuvant versus definitive chemoradiation, mostly in SCC patients, there was no survival difference between the definitive chemoradiation group and the surgical group, while higher treatment related mortality was shown in the surgical group (46). Nevertheless, in a long term analysis of the FFCD 9102 trial, higher OS was shown for clinical non-responders who underwent surgery, than those who did not (17 vs. 5.5 months, respectively) (47), suggesting efficacy for a non-operative approach in chemoradiation responders only.

The European Society for Medical Oncology guidelines indicate that definitive chemoradiation may be considered a standard alternative to esophagectomy following neoadjuvant chemoradiation in locally advanced esophageal SCC (8), while the American National Comprehensive Cancer Network guidelines recommend definitive chemoradiation in the setting of locally advanced SCC only for patients who decline surgery or those with T4b tumors (9).

\section{Targeted therapy}

To date, no significant advantage has been observed with the addition of biological agents in localized esophageal carcinoma. The monoclonal antibody against the vascular endothelial growth factor (VEGF), bevacizumab alongside the oral tyrosine kinase inhibitor erlotinib, added to preoperative chemoradiotherapy failed to improve outcomes (48). Similar negative results were observed with another monoclonal antibody directed at the epidermal growth factor receptor (EGFR) cetuximab (49). Following a positive phase I/II trial, results are anticipated for an ongoing phase III trial RTOG 1010 (50), combining the HER2-targeted agent trastuzumab with neoadjuvant chemoradiotherapy and as adjuvant treatment for one year, in patients with HER2 positive esophageal adenocarcinoma. To date, however, anti-HER2 therapy is not considered standard treatment for localized HER2-positive esophageal cancer.

\section{Metastatic disease}

Approximately $40 \%$ of patients with esophageal cancer are diagnosed with metastatic disease; with the emergence of enhanced diagnostic tools such as staging-laparoscopy, this number may in fact be even higher. Five-year survival is poor, nearly reaching $5 \%$ in most studies. Palliation and best-supportive-care are paramount in the treatment paradigm of stage IV esophageal cancer and should be provided regardless of antineoplastic treatment. Systemic therapy for metastatic adenocarcinoma follows the same principles of care as gastric adenocarcinoma, as evidence has been extrapolated from gastric/GEJ cancer studies. Encouraging phase III data has emerged and drug approvals attained (Figure 2).

\section{Chemotherapy}

Historically, the standard regimen for metastatic esophageal cancer has been the two-drug combination, CF. During the last decade, two large trials showed that capecitabine could be substituted for 5-FU and oxaliplatin for cisplatin with considerably less toxicity, in esophageal 


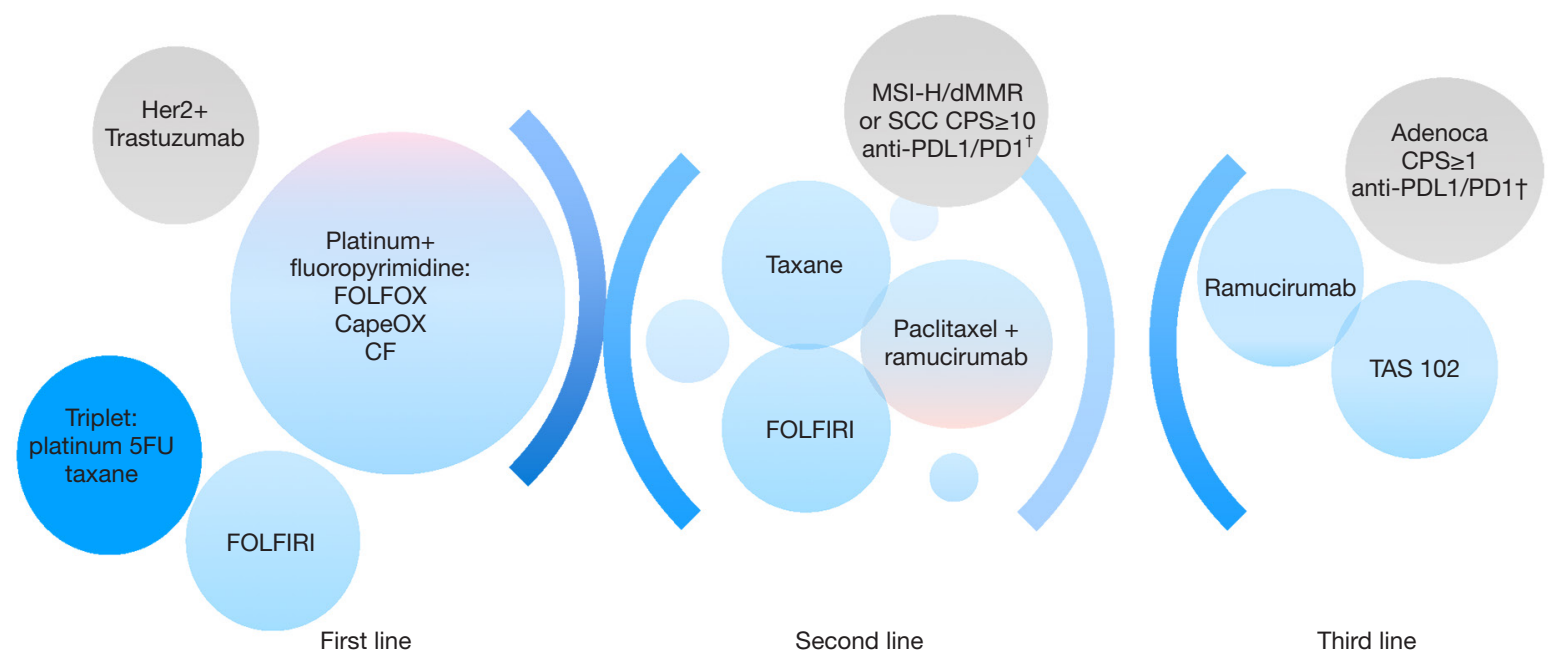

Figure 2 Treatment lines for metastatic esophageal/GEJ cancer. In the first-line setting, doublet platinum and fluoropyrimidine chemotherapy are preferred. Gray inserts indicate personalized treatment; trastuzumab for HER2+ in the first-line setting only, anti-PDL1/ PD1 for MSI-H or positive CPS. ${ }^{\dagger}$, regulatory approval gained for pembrolizumab. GEJ, gastroesophageal junction; CPS, combined positive score.

SCC and adenocarcinoma in the REAL-2 study (51), and gastroesophageal adenocarcinoma (52). Thereafter FOLFOX emerged as the most widely used first-line chemotherapy regimen worldwide. Data for FOLFIRI (fluorouracil-irinotecan-folinic acid) in gastric and GEJ tumors show non-inferiority to platinum fluoropyrimidine therapy and suggest superior time to treatment failure (53), hence this serves as an alternative protocol in case platinum drugs should be avoided.

A three-drug regimen that included the addition of docetaxel to the platinum fluoropyrimidine backbone in GEJ and gastric cancer resulted in higher response-rate and time-to-progression, with a 0.6-month improvement in median survival (54). This regimen entails higher toxicity and may be administered for medically fit patients with excellent performance status and with frequent monitoring, mainly when high response rate is warranted. Doublet regimens remain the mainstay of first-line treatment.

For patients who progress on first-line treatment and are medically fit for chemotherapy, taxanes or irinotecan are considered standard options for second-line treatment. Studies show improved symptom control and diseasespecific quality of life, however, the OS benefit of cytotoxic chemotherapy compared with best supportive care is approximately 6 weeks, as was shown for secondline docetaxel in COUGAR-02 (55). A new agent that has shown efficacy in heavily pretreated metastatic GEJ/gastric adenocarcinoma is the combined regimen of trifluridine (a nucleoside analog) and tipiracil (thymidine phosphorylase inhibitor), also known as TAS 102. TAS 102 gained regulatory approval in 2019 following the positive phase III TAGS trial that demonstrated a 2.1-month increase in OS as compared with best supportive care alone. Substantial hematological toxicity limits its administration to third line treatment of select patients with minimal symptoms and low volume disease (56).

\section{Targeted biologic agents}

A greater understanding of the genomic landscape of esophageal cancer has prompted the initiation of tailored treatment in the metastatic setting (Figure 3). Due to the similar molecular origins of adenocarcinoma of the distal esophagus, GEJ, and proximal stomach as revealed in the Cancer Genome Atlas analysis (1), data from gastric/GEJ trials is extrapolated for distal esophageal cancer as well.

\section{Human epidermal growth factor receptor 2 (HER2)}

A known driver of tumorigenesis, HER2 amplification or overexpression has been found in $7-34 \%$ of gastric cancers (57). Recent data suggest oncogenic amplification in over $30 \%$ of esophageal adenocarcinomas, while in only $3 \%$ of esophageal SCCs (1). The addition of trastuzumab to chemotherapy as compared to chemotherapy alone, was 


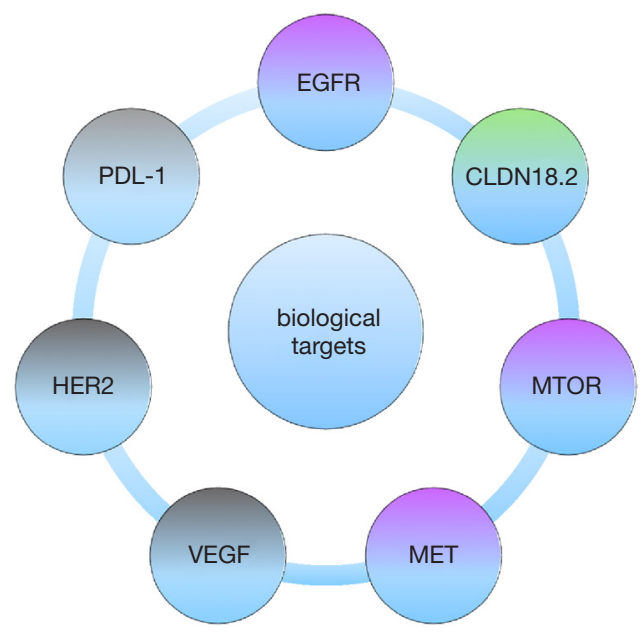

Figure 3 Major biological targets in esophageal/GEJ carcinoma. Gray tint indicates biological targets with approved therapeutic agents. Purple indicates targets with positive phase II trials that were not supported by phase III data. Green represents a target with ongoing phase III investigations. GEJ, gastroesophageal junction.

found to significantly improve all endpoints evaluated in the phase III ToGA study for HER2- positive gastric and GEJ cancer, including anti-tumor response (47.3\% vs. $34.5 \%)$, PFS (6.7 vs. 5.5 months) and OS (13.8 vs. 11.1 months)without remarkably increasing cardiac or other toxicity (57). Thereafter, trastuzumab became standard of care for firstline treatment of HER2-positive metastatic esophageal adenocarcinoma.

Significant OS benefit has not been demonstrated to date with second-line HER2 targeting agents. Lapatinib, a dual tyrosine kinase inhibitor of both HER2 and EGFR pathways, was evaluated in the TRIO-013/LOGiC study (58), and the monoclonal antibody, pertuzumab, in the $7 A C O B$ study (59); neither improved OS. Interestingly, in TyTan, a phase III Asian study, efficacy for lapatinib and paclitaxel was shown for specific subgroups: immunohistochemistry $3+$ patients compared with $0 / 1+$ and $2+$ patients, as well as Chinese compared with Japanese patients (60). No improvements in survival indices were shown with the trastuzumab conjugate antimicrotubule agent, emtansine, T-DM1 in the GATSBY study (61). The lack of success in administering anti-HER2 agents beyond progression is attributed to intra-tumoral resistance mechanisms, such as mutations or co-amplification of pathways (62).
Additionally, loss of HER2 overexpression has been identified in $16 \%$ of resistant patients (63).

Results of trials exploring two novel HER2-targeting antibodies in both gastric and breast cancer patients previously treated with HER2 inhibition are highly anticipated. Firstly, the antibody-drug conjugate DS8201 (trastuzumab deruxtecan), comprised of a humanized antibody against HER2, an enzyme-cleavable linker and a topoisomerase I inhibitor payload, has shown promising activity in a phase I trial of HER2 advanced gastric or GEJ cancer (64). Of the 44 participants, nineteen (43\%) achieved a confirmed objective response. Median duration of response lasted 7 months and OS was 12.8 months. An additional drug, ZW25, is a biparatopic antibody capable of binding two HER2 epitopes: the ECD4 (trastuzumab) and ECD2 (pertuzumab) binding domains; this unique mechanism of action is hypothesized to overcome numerous resistance mechanisms. Of the seven patients with gastroesophageal cancers in the 23-patient phase I trial, three $(43 \%)$ experienced a partial response and stable disease was observed in one patient (14\%) (65). Both novel HER2 drugs have been granted fast track designation by the food and drug administration (FDA) and the phase II DESTINY-Gastric01 and ZW25 trials are currently underway (66).

\section{VEGF}

The VEGF pathway has been actively studied in gastroesophageal carcinoma, particularly monoclonal antibodies bevacizumab and ramucirumab and the multikinase inhibitor regorafenib. Of these, only ramucirumab has attained regulatory approval, with significant OS benefit shown for patients with refractory gastric and GEJ adenocarcinoma, both as monotherapy in the REGARD trial (3.8 to 5.2 months) (67), and in conjunction with paclitaxel in the RAINBOW trial (9.6 to 7.4 months) (68). Treatment was well-tolerated besides an increase in hypertension and in the latter, more neutropenia compared to paclitaxel alone. Data has not yet shown efficacy for ramucirumab as first-line treatment. Combination with FOLFOX did not improve survival compared to FOLFOX alone (69), and the subsequent phase III RAINFALL trial investigated the addition of ramucirumab to cisplatin and a fluoropyrimidine and found improved PFS by weeks and no significant improvement in OS (70). Thereby, ramucirumab in conjunction with paclitaxel has emerged as the standard second-line therapy for metastatic GEJ adenocarcinoma. 


\section{Other emerging biological targets}

Claudin 18.2 (CLDN18.2) is a tight junction protein known to be expressed in gastric and GEJ adenocarcinoma. Zolbetuximab (IMAB362) has demonstrated activity in CLDN18.2 expressing tumor cells both as single-agent treatment and in combination with chemotherapy. The phase II study (71) included gastric/GEJ/esophageal cancer patients, and met its endpoints of improved PFS (7.9 vs. 5.7 months with or without IMAB362, respectively) and median OS (12.5 vs. 8.7), with higher expression correlating with response. Treatment was well tolerated and the phase III trial adding zolbetuximab to FOLFOX is underway (NCT03504397) (72). Despite encouraging phase II investigations, agents targeting EGFR, MTOR and MET have not yielded benefit in phase III studies (73-76).

\section{Immunotherapy}

Immunotherapy has evolved as a promising new treatment modality across cancer types, and as of lately, joined the treatment arsenal for esophageal and gastric cancer (Figure 4A,B).

\section{Pembrolizumab}

The programmed cell death protein-1 (PD-1) monoclonal antibody, pembrolizumab, was granted accelerated approval by the FDA in 2017 for the treatment of patients with unresectable or metastatic MSI-High or deficient mismatch repair (dMMR) solid tumors that have progressed on prior treatment and who have no satisfactory alternative treatment options (77). The same year, pembrolizumab first gained FDA approval for patients with recurrent, locally advanced or metastatic programmed cell death ligand-1 (PD-L1)positive gastric or GEJ adenocarcinoma who progressed on two or more prior lines of therapy. PD-L1 positivity was defined as a combined positive score (CPS) of 1 or more; this novel score method quantifies the number of PD-L1 staining tumor cells, lymphocytes and macrophages, divided by the total number of viable cells, multiplied by 100 . This first approval of pembrolizumab in esophagogastric adenocarcinoma was based on the phase Ib KEYNOTE-012 study (78) and the subsequent phase II KEYNOTE-059 (79) which showed an overall response rate of $15.5 \%$ in $\mathrm{PD}$ $\mathrm{L} 1$-positive tumors versus $6.4 \%$ in $\mathrm{PD}-\mathrm{L} 1$-negative tumors, and a median response duration of 16.3 months. Pembrolizumab failed to show significant survival benefit in second-line treatment of gastric/GEJ adenocarcinoma in the phase III KEYNOTE-061 (80) however, toxicity was reduced in comparison to chemotherapy.

Two recently published studies specifically targeted patients with esophageal cancer. The phase II KEYNOTE-180 (81) and phase III KEYNOTE-181 (82) evaluated pembrolizumab as second-line therapy for patients with advanced PD-L1positive SCC or adenocarcinoma of the esophagus and Siewert type I GEJ. Pembrolizumab significantly improved OS compared with chemotherapy when PD-L1 CPS $\geq 10$, with a more favorable safety profile. Clinically meaningful but nonsignificant survival was observed in patients with SCC and nonsignificant in the intent-to-treat population. These data led to FDA approval in second-line therapy of esophageal SCC.

Data regarding first-line treatment with pembrolizumab is currently underway. Preliminary results of an ongoing investigation of cohorts 2 and 3 of KEYNOTE-0 59 mentioned above (83-85) suggest promising activity for pembrolizumab or its combination with $\mathrm{CF}$ as firstline therapy in PD-L1-positive gastric/GEJ cancers. This combination will be further studied in the phase III KEYNOTE-590 trial (NCT03189719) (86), recruiting participants with advanced esophageal adenocarcinoma and SCC, and GEJ adenocarcinoma. Moreover, the eagerly awaited phase III KEYNOTE-062 (NCT02494583) (87) recently achieved its primary endpoint, showing that for patients with PD-L1 positive, HER2-negative, advanced gastric or GEJ cancer, first-line pembrolizumab resulted in non-inferior survival compared to standard chemotherapy. In tumors with CPS of 10 or more, OS was 17.4 months compared with 10.8 months for chemotherapy. This was maintained for a protracted period of time, with $39 \%$ of patients on pembrolizumab alive after two years compared with $22 \%$ of those on chemotherapy. In the chemotherapy plus pembrolizumab combination treatment arm, survival benefit was not shown, regardless of CPS. A limitation of the study is that the chemotherapy-only cohort rarely went on to second-line immunotherapy, whereas all patients in the other treatment arms received pembrolizumab, making it difficult to validate inferences regarding patient survival.

In conclusion, pembrolizumab is now an approved thirdline treatment option for GEJ adenocarcinoma patients with PD-L1 expression levels by CPS of 1 or more; it is also an approved second-line option for MSI-H/dMMR tumors as well as esophageal SCC with CPS of 10 or more. First-line pembrolizumab is not yet approved by regulatory bodies, but may represent an alternative for GEJ cancer with a CPS of 10 or more, as this subgroup had a profound survival benefit. For those with CPS of 1 or more, pembrolizumab should be reserved for selected patients, 
A

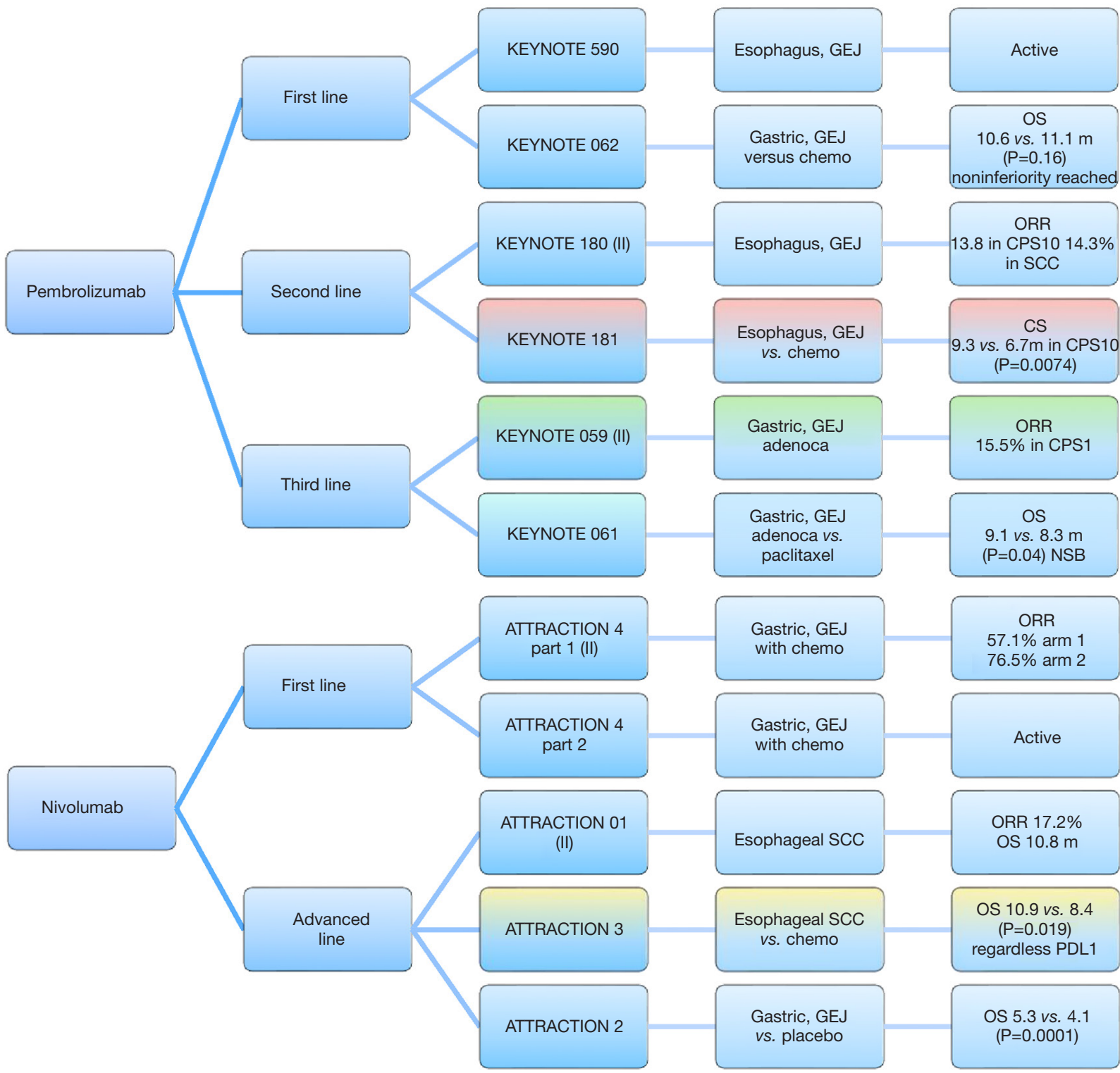

Figure 4 Immunotherapy. (A) Depiction of major trials evaluating PD1 inhibitors in esophageal/GEJ carcinoma. (A) Pembrolizumab is approved in the second and third-line setting by PDL-1 CPS criteria following KEYNOTE-181 and KEYNOTE-059, respectively, as highlighted in color. (B) Nivolumab trials have met their endpoints irrespectively of PDL-1 status. ATTRACTION-3 has shown significant results, highlighted in color. All trials are phase III unless indicated by parentheses after the study name. Phase II trials have no control arm comparator. GEJ, gastroesophageal junction.

such as frail patients for whom chemotherapy may be inappropriate, and in others, saved for later treatment lines. Combination treatment could be used in patients in need of a rapid response.

\section{Nivolumab and ipilimumab}

Another PD-1 inhibitor, nivolumab and the cytotoxic
T-lymphocyte-associated protein-4 inhibitor, ipilimumab, have also shown clinical utility in esophageal cancer, though significant results have only recently been published and not yet presented for drug approval. The phase I/II CheckMate-032 (88) found clinically meaningful activity for combination immune therapy in patients with advanced refractory esophagogastric cancer irrespective of $\mathrm{PD}$ - 
L1 status. In the larger phase III CheckMate-649 (89) for first-line treatment, the nivolumab/ipilimumab arm was terminated due to severe toxicity, while nivolumab combined with chemotherapy is ongoing.

Single agent nivolumab has been studied in the phase II ATTRACTION-01 (90) study, which enrolled Asian patients with refractory esophageal SCC and showed a steady overall response rate of $17.2 \%$ after two years, and a median duration of response of 11.17 months (91), albeit a relatively high toxicity profile. The phase III ATTRACTION-3 (92) was designed based on these positive results in refractory SCC and enrolled patients from a variety of geographic locations. A significant OS advantage was recently shown across subgroups irrespective of PD-L1 expression, for second-line nivolumab compared with chemotherapy (10.9 vs. 8.4 months, respectively), alongside a favorable safety profile. While overall responses were similar (19\% vs. 22\%), duration of response was longer with nivolumab (6.9 vs. 3.9 months).

Nivolumab for heavily pretreated gastric/GEJ cancer was also found to significantly improve OS (5.26 vs. 4.14 months in the placebo arm) in an Asian population, in the phase III ATTRACTION-2 (93) trial, which was maintained over time and associated with reduced toxicity. Separate subgroup analyses found that nivolumab was effective regardless of prior trastuzumab (94) or ramucirumab (95) use, and that response and progression free survival were numerically higher in those with prior ramucirumab use than in those without. Finally, first-line nivolumab in combination with capecitabine and oxaliplatin chemotherapy in advanced gastric/GEJ cancer is currently being evaluated in the phase III ATTRACTION-4 (96) study, following positive phase II results.

These data support nivolumab use in second-line treatment of esophageal SCC and GEJ cancer, regardless of PD-L1 expression.

\section{Challenges}

As treatment of esophageal cancer improves, new challenges and obstacles must be addressed in order to further advance the field and improve patient outcomes. Three current controversies will be discussed.

\section{Preferred approach for localized disease}

Determining superiority of treatment approach is a matter of active dispute in the localized disease setting. This is still no mature head-to-head evidence comparing neoadjuvant chemoradiation with perioperative chemotherapy. An ongoing trial in Germany, ESOPEC (NCT92509286) (97), is evaluating the CROSS and FLOT regimens without radiotherapy, head-to-head, and may shed light on the preferred approach. Moreover, Neo-AEGIS ICORG 10-14 (NCT01726452) (98) will assess CROSS versus pre and postoperative chemotherapy with ECF/ECX and TOPGEAR (NCT01924819) (99), preoperative chemotherapy with ECF, with or without subsequent chemoradiation.

\section{Standardization of radiation}

Another unsolved issue in the neoadjuvant setting remains defining a uniform radiation dose. The CROSS study used 41.4 Gy, while the commonly accepted dose is 50.4 Gy at $1.8 \mathrm{~Gy}$ per fraction, as employed in several prospective trials. The German Oesophageal Cancer Study Group and others used a dose-escalated regimen of up to $60 \mathrm{~Gy}$, as non-operative care, an approach currently evaluated in the SCOPE2 trial (NCT02741856) (100). Optimization of radiation techniques in the modern era, with utilization of three-dimensional conformal radiation therapy, intensitymodulated radiation therapy (IMRT), protons, and rigorous fractionation schedules, have greatly improved outcomes, with reduced toxicity and comparable survival rates (6). Chemotherapy with IMRT has not been compared to equivalent chemotherapy alongside started $3 \mathrm{D}$ radiotherapy.

\section{Targeted therapy in advanced lines}

Response to anti-HER2 therapies in advanced lines of care constitutes a biological challenge. In breast cancer, HER2 positive tumors remain responsive to successive antiHER2 therapy after progression on first-line trastuzumab, enabling the incorporation of a wide arsenal of anti-HER2 agents. Unlike breast cancer, HER2-positive gastric/gastroesophageal cancer follows a differential biological course, as acquired resistance following first-line anti-HER2 treatment prevails, significantly reducing responses to further anti-HER2 therapy. The evolution of liquid biopsies and extensive basic research may eventually reveal surrogate biomarkers to effectively select candidates for advanced lines of anti-HER2 therapy, as well as measures to regain sensitivity to anti-HER2 agents.

\section{Conclusions}

A heterogenous disease distinguished by tumor location, 
histology and biology, esophageal cancer has entered the forefront of oncological research. As its genomic landscape is being elucidated and diverse treatment approaches explored, management is becoming increasingly tailored to disease traits and the host mechanisms. Ideally, a combination of chemotherapy, radiation, surgery, biologic therapy and palliation is determined by a multidisciplinary team prior to treatment onset and reevaluated throughout. Taken together, with the aim of improving patient outcomes and pushing their survival curves, these evolving paradigms ultimately bring about a new era in esophageal cancer care.

\section{Acknowledgments}

Funding: None.

\section{Footnote}

Conflicts of Interest: Both authors have completed the ICMJE uniform disclosure form (available at http://dx.doi. org/10.21037/atm.2020.03.110). The authors have no conflicts of interest to declare.

Ethical Statement: The authors are accountable for all aspects of the work in ensuring that questions related to the accuracy or integrity of any part of the work are appropriately investigated and resolved.

Open Access Statement: This is an Open Access article distributed in accordance with the Creative Commons Attribution-NonCommercial-NoDerivs 4.0 International License (CC BY-NC-ND 4.0), which permits the noncommercial replication and distribution of the article with the strict proviso that no changes or edits are made and the original work is properly cited (including links to both the formal publication through the relevant DOI and the license). See: https://creativecommons.org/licenses/by-nc-nd/4.0/.

\section{References}

1. Cancer Genome Atlas Research Network; Analysis Working Group: Asan University; BC Cancer Agency; Brigham and Women's Hospital; Broad Institute; et al. Integrated genomic characterization of oesophageal carcinoma. Nature 2017;541:169-75.

2. Gibson M. Epidemiology and pathobiology of esophageal cancer. Post TW, ed. UpToDate. Waltham, MA: UpToDate Inc. Available online: https://www.uptodate. com (accessed on November 02, 2019).

3. Bray F, Ferlay J, Soerjomataram I, Siegel RL, Torre LA, Jemal A. Global cancer statistics 2018: GLOBOCAN estimates of incidence and mortality worldwide for 36 cancers in 185 countries. CA Cancer J Clin 2018;68:394-424.

4. Cancer Stat Facts: Esophageal Cancer. Originally published by the National Cancer Institute. Available online: https://seer.cancer.gov/statfacts/html/esoph.html. Accessed November 2, 2019.

5. Kelsen DP, Ginsberg R, Pajak TF, et al. Chemotherapy followed by surgery compared with surgery alone for localized esophageal cancer. N Engl J Med 1998;339:1979.

6. Sun XD, Yu JM, Fan XL, Ren RM, Li MH, Zhang GL. Randomized clinical study of surgery versus radiotherapy alone in the treatment of resectable esophageal cancer in the chest. Zhonghua Zhong Liu Za Zhi 2006;28:784-7.

7. Rice TW, Rusch VW, Apperson-Hansen C, et al. Worldwide esophageal cancer collaboration. Dis Esophagus 2009;22:1.

8. Lordick F, Mariette C, Haustermans K, et al. Oesophageal cancer: ESMO Clinical Practice Guidelines for diagnosis, treatment and follow-up. Ann Oncol 2016;27:v50-v57.

9. National Comprehensive Cancer Network. Esophageal and Esophagogastric Junction Cancers (Version 4.2019). Available online: https://www.nccn.org/professionals/ physician_gls/ pdf/esophageal.pdf. Accessed December 30, 2019.

10. Gaast AV, P. van Hagen, M. Hulshof et al. Effect of preoperative concurrent chemoradiotherapy on survival of patients with resectable esophageal or esophagogastric cancer: Results from a multinational randomized phase III study (abstract 4004). J Clin Oncol 2010;28:302s.

11. Tepper J, Krasna MJ, Niedzwiecki D, et al. Phase III trial of trimodality therapy with cisplatin, fluorouracil, radiotherapy, and surgery compared with surgery alone for esophageal cancer: CALGB 9781. J Clin Oncol 2008;26:1086-92.

12. Yang H, Liu H, Chen $\mathrm{Y}$, et al. Neoadjuvant chemoradiotherapy followed by surgery versus surgery alone for locally advanced squamous cell carcinoma of the esophagus (NEOCRTEC5010): a phase III multicenter, randomized, open-label clinical trial. J Clin Oncol 2018;36:2796-803.

13. Shapiro J, van Lanschot JJB, Hulshof M, et al. Neoadjuvant chemoradiotherapy plus surgery versus surgery alone for oesophageal or junctional cancer (CROSS): long-term results of a randomised controlled 
trial. Lancet Oncol 2015;16:1090-8.

14. Chan KKW, Saluja R, Delos Santos K, et al. Neoadjuvant treatments for locally advanced, resectable esophageal cancer: A network meta-analysis. Int J Cancer 2018;143:430.

15. Pasquali S, Yim G, Vohra RS, et al. Survival After Neoadjuvant and Adjuvant Treatments Compared to Surgery Alone for Resectable Esophageal Carcinoma: A Network Meta-analysis. Ann Surg 2017;265:481.

16. Sjoquist KM, Burmeister BH, Smithers BM, et al. Survival after neoadjuvant chemotherapy or chemoradiotherapy for resectable oesophageal carcinoma: an updated metaanalysis. Lancet Oncol 2011;12:681.

17. Leichman LP, Goldman BH, Bohanes PO, et al. S0356: a phase II clinical and prospective molecular trial with oxaliplatin, fluorouracil, and external-beam radiation therapy before surgery for patients with esophageal adenocarcinoma. J Clin Oncol 2011;29:4555-60.

18. Messager M, Mirabel X, Tresch E, et al. Preoperative chemoradiation with paclitaxel-carboplatin or with fluorouracil-oxaliplatin-folinic acid (FOLFOX) for resectable esophageal and junctional cancer: the PROTECT-1402, randomized phase 2 trial. BMC Cancer 2016;16:318.

19. Cunningham D, Allum WH, Stenning SP, et al. Perioperative chemotherapy versus surgery alone for resectable gastroesophageal cancer. $\mathrm{N}$ Engl J Med 2006;355:11-20.

20. Al-Batran SE, Homann N, Pauligk C, et al. Perioperative chemotherapy with fluorouracil plus leucovorin, oxaliplatin, and docetaxel versus fluorouracil or capecitabine plus cisplatin and epirubicin for locally advanced, resectable gastric or gastro-oesophageal junction adenocarcinoma (FLOT4): a randomised, phase 2/3 trial. Lancet 2019;393:1948-57.

21. Al-Batran SE, Hofheinz RD, Pauligk C, et al.

Histopathological regression after neoadjuvant docetaxel, oxaliplatin, fluorouracil, and leucovorin versus epirubicin, cisplatin, and fluorouracil or capecitabine in patients with resectable gastric or gastro-oesophageal junction adenocarcinoma (FLOT4-AIO): results from the phase 2 part of a multicentre, open-label, randomised phase 2/3 trial. Lancet Oncol 2016;17:1697-708.

22. Ychou M, Boige V, Pignon JP, et al. Perioperative chemotherapy compared with surgery alone for resectable gastroesophageal adenocarcinoma: an FNCLCC and FFCD multicenter phase III trial. J Clin Oncol 2011;29:1715-21.
23. Medical Research Council Oesophageal Cancer Working Group. Surgical resection with or without preoperative chemotherapy in oesophageal cancer: a randomised controlled trial. Lancet 2002;359:1727-33.

24. Allum WH, Stenning SP, Bancewicz J, et al. Long-term results of a randomized trial of surgery with or without preoperative chemotherapy in esophageal cancer. J Clin Oncol 2009;27:5062-7.

25. Alderson D, Cunningham D, Nankivell M, et al. Neoadjuvant cisplatin and fluorouracil versus epirubicin, cisplatin, and capecitabine followed by resection in patients with oesophageal adenocarcinoma (UK MRC OE05): an open-label, randomised phase 3 trial. Lancet Oncol 2017;18:1249-60.

26. Stahl M, Walz MK, Stuschke M, et al. Phase III comparison of preoperative chemotherapy compared with chemoradiotherapy in patients with locally advanced adenocarcinoma of the esophagogastric junction. J Clin Oncol 2009;27:851-6.

27. Ilson DH, Minsky BD, Ku GY, et al. Phase 2 trial of induction and concurrent chemoradiotherapy with weekly irinotecan and cisplatin followed by surgery for esophageal cancer. Cancer 2012;118:2820-7.

28. Ajani JA, Xiao L, Roth JA, et al. A phase II randomized trial of induction chemotherapy versus no induction chemotherapy followed by preoperative chemoradiation in patients with esophageal cancer. Ann Oncol 2013;24:2844-9.

29. Ruhstaller T, Widmer L, Schuller JC, et al. Multicenter phase II trial of preoperative induction chemotherapy followed by chemoradiation with docetaxel and cisplatin for locally advanced esophageal carcinoma (SAKK 75/02). Ann Oncol 2009;20:1522-8.

30. Macdonald JS, Smalley SR, Benedetti J, et al. Chemoradiotherapy after surgery compared with surgery alone for adenocarcinoma of the stomach or gastroesophageal junction. N Engl J Med 2001;345:725-30.

31. Smalley SR, Benedetti JK, Haller DG, et al. Updated analysis of SWOG-directed intergroup study 0116: a phase III trial of adjuvant radiochemotherapy versus observation after curative gastric cancer resection. J Clin Oncol 2012;30:2327-33.

32. Adelstein DJ, Rice TW, Rybicki LA, et al. Mature results from a phase II trial of postoperative concurrent chemoradiotherapy for poor prognosis cancer of the esophagus and gastroesophageal junction. J Thorac Oncol 2009;4:1264-9.

33. Kang J, Chang JY, Sun X, Men Y, Zeng H, Hui Z. Role 
of Postoperative Concurrent Chemoradiotherapy for Esophageal Carcinoma: A meta-analysis of 2165 Patients. J Cancer 2018;9:584-93.

34. Kelsen DP, Winter KA, Gunderson LL, et al. Longterm results of RTOG trial 8911 (USA Intergroup 113): a random assignment trial comparison of chemotherapy followed by surgery compared with surgery alone for esophageal cancer. J Clin Oncol 2007;25:3719-25.

35. Cats A, Jansen EPM, Grieken NCT et al. Chemotherapy versus chemoradiotherapy after surgery and preoperative chemotherapy for resectable gastric cancer (CRITICS): an international, open-label, randomised phase 3 trial. Lancet Oncol 2018;19:616-28.

36. Noh SH, Park SR, Yang HK, et al. Adjuvant capecitabine plus oxaliplatin for gastric cancer after D2 gastrectomy (CLASSIC): 5-year follow-up of an open-label, randomised phase 3 trial. Lancet Oncol 2014;15:1389-96.

37. Wong R, Malthaner R. Combined chemotherapy and radiotherapy (without surgery) compared with radiotherapy alone in localized carcinoma of the esophagus. Cochrane Database Syst Rev 2006;25:CD002092.

38. Herskovic A, Martz K, al-Sarraf M, et al. Combined chemotherapy and radiotherapy compared with radiotherapy alone in patients with cancer of the esophagus. N Engl J Med 1992;326:1593-8.

39. Cooper JS, Guo MD, Herskovic A, et al. Chemoradiotherapy of locally advanced esophageal cancer: long-term follow-up of a prospective randomized trial (RTOG 85-01). Radiation Therapy Oncology Group. JAMA 1999;281:1623-7.

40. al-Sarraf M, Martz K, Herskovic A. et al. Progress report of combined chemoradiotherapy versus radiotherapy alone in patients with esophageal cancer: an intergroup study. J Clin Oncol 1997;15:277-84.

41. Minsky BD, Pajak TF, Ginsberg RJ, et al. INT 0123 (Radiation Therapy Oncology Group 94-05) phase III trial of combined-modality therapy for esophageal cancer: high-dose versus standard-dose radiation therapy. J Clin Oncol 2002;20:1167-74.

42. Conroy T, Galais MP, Raoul JL, et al. Definitive chemoradiotherapy with FOLFOX versus fluorouracil and cisplatin in patients with oesophageal cancer (PRODIGE5/ ACCORD17): final results of a randomised, phase 2/3 trial. Lancet Oncol 2014;15:305-14.

43. Li QQ, Liu MZ, Hu YH, et al. Definitive concomitant chemoradiotherapy with docetaxel and cisplatin in squamous esophageal carcinoma. Dis Esophagus 2010;23:253-9.
44. Alexandrou A, Davis PA, Law S, et al. Squamous cell carcinoma and adenocarcinoma of the lower third of the esophagus and gastric cardia: similarities and differences. Dis Esophagus 2002;15:290.

45. Koppert LB, Janssen-Heijnen ML, Louwman MW, et al. Comparison of comorbidity prevalence in oesophageal and gastric carcinoma patients: a population-based study. Eur J Gastroenterol Hepatol 2004;16:681.

46. Vellayappan BA, Soon YY, Ku GY, et al. Chemoradiotherapy versus chemoradiotherapy plus surgery for esophageal cancer. Cochrane Database Syst Rev 2017;8:CD010511.

47. Vincent J, Mariette C, Pezet D, et al. Early surgery for failure after chemoradiation in operable thoracic oesophageal cancer. Analysis of the non-randomised patients in FFCD 9102 phase III trial: Chemoradiation followed by surgery versus chemoradiation alone. Eur J Cancer 2015;51:1683-93.

48. Bendell JC, Meluch A, Peyton J, et al. A phase II trial of preoperative concurrent chemotherapy/radiation therapy plus bevacizumab/erlotinib in the treatment of localized esophageal cancer. Clin Adv Hematol Oncol 2012;10:430-7.

49. Suntharalingam M, Winter K, Ilson D, et al. Effect of the Addition of Cetuximab to Paclitaxel, Cisplatin, and Radiation Therapy for Patients With Esophageal Cancer. The NRG Oncology RTOG 0436 Phase 3 Randomized Clinical Trial. JAMA Oncol 2017;3:1520-8.

50. A Phase III Trial Evaluating the Addition of Trastuzumab to Trimodality Treatment of HER2-Overexpressing Esophageal Adenocarcinoma (NCT01196390). Available online: https://clinicaltrials.gov/ct2/show/NCT01196390

51. Cunningham D, Starling N, Rao S, et al. Capecitabine and oxaliplatin for advanced esophagogastric cancer. N Engl J Med 2008;358:36-46.

52. Al-Batran SE, Hartmann JT, Probst S, et al. Phase III trial in metastatic gastroesophageal adenocarcinoma with fluorouracil, leucovorin plus either oxaliplatin or cisplatin: a study of the Arbeitsgemeinschaft Internistische Onkologie. J Clin Oncol 2008;26:1435-42.

53. Guimbaud R, Louvet C, Ries P, et al. Prospective, randomized, multicenter, phase III study of fluorouracil, leucovorin, and irinotecan versus epirubicin, cisplatin, and capecitabine in advanced gastric adenocarcinoma: A French Intergroup (Fédération Francophone de Cancérologie Digestive, Fédération Nationale des Centres de Lutte Contre le Cancer, and Groupe Coopérateur Multidisciplinaire en ncologie) Study. J Clin Oncol 
2014;32:3520-6.

54. Van Cutsem E, Moiseyenko VM, Tjulandin S, et al. Phase III study of docetaxel and cisplatin plus fluorouracil compared with cisplatin and fluorouracil as first-line therapy for advanced gastric cancer: a report of the V325 Study Group. J Clin Oncol 2006;24:4991-7.

55. Ford HE, Marshall A, Bridgewater JA, et al. Docetaxel versus active symptom control for refractory oesophagogastric adenocarcinoma (COUGAR-02): an open-label, phase 3 randomised controlled trial. Lancet Oncol 2014;15:78-86.

56. Shitara K, Doi T, Dvorkin M, et al. Trifluridine/tipiracil versus placebo in patients with heavily pretreated metastatic gastric cancer (TAGS): a randomised, doubleblind, placebo-controlled, phase 3 trial. Lancet Oncol 2018;19:1437-48.

57. Bang YJ, Van Cutsem E, Feyereislova A, et al. Trastuzumab in combination with chemotherapy versus chemotherapy alone for treatment of HER2-positive advanced gastric or gastro- oesophageal junction cancer (ToGA): a phase 3, open-label, randomised controlled trial. Lancet 2010;376:687-97.

58. Hecht JR, Bang YJ, Qin SK, et al. Lapatinib in Combination With Capecitabine Plus Oxaliplatin in Human Epidermal Growth Factor Receptor 2-Positive Advanced or Metastatic Gastric, Esophageal, or Gastroesophageal Adenocarcinoma: TRIO-013/ LOGiC--A Randomized Phase III Trial. J Clin Oncol 2016;34:443-51.

59. Tabernero J, Hoff PM, Shen L et al. Pertuzumab plus trastuzumab and chemotherapy for HER2-positive metastatic gastric or gastro-oesophageal junction cancer (JACOB): final analysis of a double-blind, randomised, placebo-controlled phase 3 study. Lancet Oncol 2018;19:1372-84.

60. Satoh T, Xu RH, Chung HC, et al. Lapatinib plus paclitaxel versus paclitaxel alone in the second-line treatment of HER2-amplified advanced gastric cancer in Asian populations: TyTAN--a randomized, phase III study. J Clin Oncol 2014;32:2039-49.

61. Thuss-Patience PC, Shah MA, Ohtsu A, et al. Trastuzumab emtasine versus taxane use for previously treated HER2positive locally advanced or metastatic gastric or gastrooesophageal junction adenocarcinoma (GATSBY): an international randomised, open-label, adaptive, phase $2 / 3$ study. Lancet Oncol 2017;18:640-53.

62. Kim J, Fox C, Peng S, et al. Preexisting oncogenic events impact trastuzumab sensitivity in ERBB2- amplified gastroesophageal adenocarcinoma. J Clin Invest 2014;124:5145-58.

63. Janjigian YY, Sanchez-Vega F, Jonsson P, et al. Genetic predictors of response to systemic therapy in esophagogastric cancer. Cancer Discov 2018;8:49-58.

64. Shitara K, Iwata H, Takahashi S, et al. Trastuzumab deruxtecan (DS-8201a) in patients with advanced HER2positive gastric cancer: a dose-expansion, phase 1 study. Lancet Oncol 2019;20:827-36.

65. Meric-Bernstam F, Beeram M, Mayordomo JI, et al. Single agent activity of ZW25, a HER2-targeted bispecific antibody, in heavily pretreated HER2-expressing cancers. J Clin Oncol 2018;36:abstr 2500.

66. Zymeworks' lead asset, ZW25, granted Fast Track designation from the FDA [news release]. Vancouver, BC; Zymeworks Inc.: May 29, 2019. Available online: https:// bit.ly/2Mt6iJQ. Accessed October 30, 2019.

67. Fuchs CS, Tomasek J, Yong CJ, et al. Ramucirumab monotherapy for previously treated advanced gastric or gastro-oesophageal junction adenocarcinoma (REGARD): an international, randomised, multicentre, placebocontrolled, phase 3 trial. Lancet 2014;383:31-9.

68. Wilke H, Muro K, Van Cutsem E, et al. Ramucirumab plus paclitaxel versus placebo plus paclitaxel in patients with previously treated advanced gastric or gastro-oesophageal junction adenocarcinoma (RAINBOW): a double-blind, randomised phase 3 trial. Lancet Oncol 2014;15:1224-35.

69. Yoon HH, Bendell JC, Braiteh FS, et al. Ramucirumab combined with FOLFOX as front-line therapy for advanced esophageal, gastroesophageal junction, or gastric adenocarcinoma: a randomized, double-blind, multicenter Phase II trial. Ann Oncol 2016;27:2196-203.

70. Fuchs CS, Shitara K, Di Bartolomeo M, et al. Ramucirumab with cisplatin and fluoropyrimidine as first-line therapy in patients with metastatic gastric or junctional adenocarcinoma (RAINFALL): a double-blind, randomised, placebo-controlled, phase 3 trial. Lancet Oncol 2019;20:420-35.

71. Schuler M, Al-Batran S, Zvirbule Z, et al. Final results of the FAST study, an international, multicenter, randomized, phase II trial of epirubicin, oxaliplatin, and capecitabine (EOX) with or without the anti-CLDN18.2 antibody IMAB362 as first-line therapy in patients with advanced CLDN18.2+ gastric and gastroesophageal junction (GEJ) adenocarcinoma. Ann Oncol 2016;27:vi218.

72. A Phase 3 Efficacy, Safety and Tolerability Study of Zolbetuximab (Experimental Drug) Plus mFOLFOX6 Chemotherapy Compared to Placebo Plus mFOLFOX6 
as Treatment for Gastric and Gastroesophageal Junction (GEJ) Cancer (Spotlight). Available online: https:// clinicaltrials.gov/ct2/show/NCT03504397

73. Lordick F, Kang YK, Chung HC, et al. Capecitabine and cisplatin with or without cetuximab for patients with previously untreated advanced gastric cancer (EXPAND): a randomised, open-label phase 3 trial. Lancet Oncol 2013;14:490-9.

74. Waddell T, Chau I, Cunningham D, et al. Epirubicin, oxaliplatin, and capecitabine with or without panitumumab for patients with previously untreated advanced oesophagogastric cancer (REAL3): a randomised, openlabel phase 3 trial [published correction appears in Lancet Oncol. 2013;14:e254. Frances, Alicia [corrected to Okines, Alicia Frances Clare]. Lancet Oncol 2013;14:481-9.

75. Ohtsu A, Ajani JA, Bai YX, et al. Everolimus for previously treated advanced gastric cancer: results of the randomized, double-blind, phase III GRANITE-1 study. J Clin Oncol 2013;31:3935-43.

76. Shah MA, Bang YJ, Lordick F, et al. Effect of Fluorouracil, Leucovorin, and Oxaliplatin With or Without Onartuzumab in HER2-Negative, MET-Positive Gastroesophageal Adenocarcinoma: The METGastric Randomized Clinical Trial. JAMA Oncol 2017;3:620-7.

77. U.S. Food and Drug Administration. FDA grants accelerated approval to pembrolizumab for first tissue/ site agnostic indication. 2017. Available at: Available online: https://www.accessdata.fda.gov/drugsatfda_docs/ label/2017/125514s014lbl.pdf. Accessed Jan 4, 2020.

78. Muro K, Chung HC, Shankaran V, et al. Pembrolizumab for patients with PD-L1-positive advanced gastric cancer (KEYNOTE-012): a multicentre, open-label, phase 1b trial. Lancet Oncol 2016;17:717-26.

79. Fuchs CS, Doi T, Jang RW, et al. Safety and efficacy of pembrolizumab monotherapy in patients with previously treated advanced gastric and gastroesophageal junction cancer: phase 2 clinical KEYNOTE- 059 trial. JAMA Oncol 2018;4:e180013.

80. Shitara K, Özgüroglu M, Bang Y-J, et al. Pembrolizumab versus paclitaxel for previously treated, advanced gastric or gastro-oesophageal junction cancer (KEYNOTE-061): a randomised, open-label, controlled, phase 3 trial. Lancet 2018;392:123-33.

81. Shah MA, Kojima T, Hochhauser D, et al. Efficacy and safety of pembrolizumab for heavily pretreated patients with advanced, metastatic adenocarcinoma or squamous cell carcinoma of the esophagus: the phase 2 KEYNOTE-180 study. JAMA Oncol 2019;5:546-50.
82. Kojima T, Muro K, Francois E, et al. Pembrolizumab versus chemotherapy as second-line therapy for advanced esophageal cancer: phase III KEYNOTE-181 study. J Clin Oncol 2019;37:2.

83. Bang YJ, Muro K, Fuchs CS, et al. KEYNOTE-059 cohort 2: safety and efficacy of pembrolizumab (pembro) plus 5-fluorouracil (5-FU) and cisplatin for first-line (1L) treatment of advanced gastric cancer. J Clin Oncol 2017;35:4012.

84. Wainberg ZA, Jalal S, Muro K, et al. KEYNOTE-059 update: efficacy and safety of pembrolizumab alone or in combination with chemotherapy in patients with advanced gastric or gastroesophageal (G/GEJ) cancer. Ann Oncol 2017;28:v605-v649.

85. Catenacci DV, Wainberg Z, Fuchs CS, et al. KEYNOTE-059 cohort 3: safety and efficacy of pembrolizumab monotherapy for first-line treatment of patients (pts) with PD-L1-positive advanced gastric/gastroesophageal (G/GEJ) cancer. Ann Oncol 2017;28:suppl_3.

86. First-line Esophageal Carcinoma Study With Chemo vs. Chemo Plus Pembrolizumab (MK-3475-590/ KEYNOTE-590). Available online: https://clinicaltrials. gov/ct2/show/NCT03189719

87. Josep Tabernero, Eric Van Cutsem, Yung-Jue Bang, et al. Pembrolizumab with or without chemotherapy versus chemotherapy for advanced gastric or gastroesophageal junction (G/GEJ) adenocarcinoma: The phase III KEYNOTE-062 study. Available online: https:// meetinglibrary.asco.org/record/173187/abstract

88. Janjigian YY, Bendell J, Calvo E, et al. CheckMate-032 study: efficacy and safety of nivolumab and nivolumab plus ipilimumab in patients with metastatic esophagogastric cancer. J Clin Oncol 2018;36:2836-44.

89. Moehler MH, Janjigian YY, Adenis A, et al. CheckMate 649: A randomized, multicenter, open-label, phase III study of nivolumab (NIVO) + ipilimumab (IPI) or nivo + chemotherapy $(\mathrm{CTX})$ versus CTX alone in patients with previously untreated advanced (Adv) gastric $(\mathrm{G})$ or gastroesophageal junction (GEJ) cancer. J Clin Oncol 2018;36:192.

90. Kudo T, Hamamoto Y, Kato K, et al. Nivolumab treatment for oesophageal squamous-cell carcinoma: an open-label, multicentre, phase 2 trial. Lancet Oncol 2017;18:631-9.

91. Kitagawa Y, Doki Y, Kato K, et al. Two year survival and safety update for esophageal squamous cell carcinoma treated with nivolumab (ATTRACTION-01/ONO-4538- 
07), Ann Onc 2017;28:mdx369.022.

92. Kato K, Cho BC, Takahashi M, et al. Nivolumab versus chemotherapy in patients with advanced oesophageal squamous cell carcinoma refractory or intolerant to previous chemotherapy (ATTRACTION-3): a multicentre, randomised, open-label, phase 3 trial. Lancet Oncol 2019;20:1506-17.

93. Kang YK, Boku N, Satoh T, et al. Nivolumab in patients with advanced gastric or gastro-oesophageal junction cancer refractory to, or intolerant of, at least two previous chemotherapy regimens (ONO-4538- 12, ATTRACTION-2): a randomised, double-blind, placebocontrolled, phase 3 trial. Lancet 2017;390:2461-71.

94. Satoh T, Kang Y, Chao Y, et al. Exploratory subgroup analysis of patients with prior trastuzumab use in the ATTRACTION-2 trial: a randomized phase III clinical trial investigating the efficacy and safety of nivolumab in patients with advanced gastric/gastroesophageal junction cancer. Gastric Cancer 2020;23:143-53.

95. Kato K, Satoh T, Muro K, et al. A subanalysis of Japanese patients in a randomized, double-blind, placebo-controlled, phase 3 trial of nivolumab for patients with advanced gastric or gastro-esophageal junction cancer refractory to, or intolerant of, at least two previous chemotherapy regimens (ONO-4538-12, ATTRACTION-2). Gastric Cancer 2019;22:344.

96. Boku N, Ryu MH, Kato K, et al. Safety and efficacy of nivolumab in combination with $\mathrm{S}-1$ /capecitabine

Cite this article as: Turgeman I, Ben-Aharon I. Evolving treatment paradigms in esophageal cancer. Ann Transl Med 2021;9(10):903. doi: 10.21037/atm.2020.03.110 plus oxaliplatin in patients with previously untreated, unresectable, advanced, or recurrent gastric/ gastroesophageal junction cancer: interim results of a randomized, phase II trial (ATTRACTION-4). Ann Oncol 2019;30:250-8.

97. Hoeppner J, Lordick F, Brunner T, et al. ESOPEC: prospective randomized controlled multicenter phase III trial comparing perioperative chemotherapy (FLOT protocol) to neoadjuvant chemoradiation (CROSS protocol) in patients with adenocarcinoma of the esophagus (NCT02509286). BMC Cancer 2016;16:503.

98. Keegan N, Keane F, Cuffe S, et al. ICORG 10-14: NeoAEGIS: A randomized clinical trial of neoadjuvant and adjuvant chemotherapy (modified MAGIC regimen) versus neoadjuvant chemoradiation (CROSS protocol) in adenocarcinoma of the esophagus and esophagogastric junction. ASCO Annual Meeting Proceedings, 2014.

99. Leong T, Smithers BM, Michael M, et al. TOPGEAR: a randomised phase III trial of perioperative ECF chemotherapy versus preoperative chemoradiation plus perioperative ECF chemotherapy for resectable gastric cancer (an international, intergroup trial of the AGITG/TROG/EORTC/NCIC CTG). BMC Cancer 2015;15:532.

100. Study of Chemoradiotherapy in Oesophageal Cancer Including PET Response and Dose Escalation (SCOPE2). Available online: https://clinicaltrials.gov/ct2/show/ NCT02741856 